\title{
Unsteady Aerodynamics of Pitching Low Aspect Ratio Wings: A review of AVT 202 panel results (Invited Paper)
}

\author{
Luis P. Bernal ${ }^{1}$ \\ University of Michigan, Ann Arbor, MI, 48109, U.S.A.
}

\begin{abstract}
This paper is an overview of research results from the NATO-STO AVT 202 panel on "Extensions of Fundamental Flow Physics to Practical MAV Aerodynamics." The focus is on the unsteady aerodynamics of pitching low aspect ratio wings. We present results from participating teams and discuss relevant flow physics. We consider translating and rotating pitching wings. The pitch motion is from 0 to $45^{\circ}$ angle of attack and two pitch rates are considered: A fast pitch rate in which the pitch motion occurs over one convective time and a slow pitch rate in which the pitch motion is over 6 convective times. Unsteady force measurement, flow visualization amd PIV measurements of the velocity and vorticity fields were obtained by AVT 202 team members. Here we focus on unsteady force measurements and flow visualization results of the LEV, TEV and TV vortices.
\end{abstract}

\section{Introduction}

$\mathrm{T}$ his paper summarizes the results and conclusions of the NATO Science and Technology Office AVT 202 panel "Extension of Fundamental Flow Physics to Practical MAV Aerodynamics ${ }^{1 "}$ on translating and rotating pitching wings. The panel report includes a detailed account of the results and conclusions. Rapidly pitching wings is a canonical unsteady aerodynamics problem that encompasses flow physics relevant to maneuvering aircraft, flapping wing systems and rotorcraft aerodynamics. For large amplitude pitch motions the flow physics include large-scale separation and concomitant vortical flow development. The emphasis of this work is on small air vehicles that operate at low speeds and therefore low Reynolds numbers. At these conditions, wing motion time scales can be comparable to relevant aerodynamic time scales, and unsteady flow phenomena are an important contribution to the force history and vortical flow development. The approach is to focus on two wing geometries and wing motion kinematics. Panel team members conducted detail experimental and numerical studies of nominally the same flow, and the results compared to elucidate the relevant flow physics. A few team members also conducted flow visualization and PIV measurements, and the results of these experiments are presented here.

When a lifting wing is set in motion from rest, it produces a starting vortex at the trailing edge or a trailing edge vortex (TEV). The TEV forms with vorticity generally parallel to the trailing edge. Also at the wing tips, flow around the tip produces a tip vortex (TV) with vorticity predominantly aligned with the flow direction. These vortices form for attached and separated flow. If the flow about the wing separates, a vortex may form at the leading edge (LEV). One important goal of the AVT 202 panel is to gain a better understanding of the vortical flow structure during unsteady pitching motions and provide qualitative and quantitative data on vortex development. The time scales structure of the LEV, TEV and TV are not understood and are one of the motivations for the research.

\section{A. Wing Motion Kinematics}

We consider the pitching motion about the leading edge of rectangular wings from zero to $45^{\circ}$ at two constant pitching rates. Figure 1 illustrates the angle of incidence as a function of time. The time axis is normalized by the convective time (chord/free stream speed), and therefore represents distance traveled by the free stream flow, or in

\footnotetext{
${ }^{1}$ Professor, Department of Aerospace Engineering, University of Michigan, 1 pb@umich.edu, and AIAA Senior Member.
} 
the frame of reference moving with the wing, distance traveled by the wing. We consider two cases: the "fast pitch case" in which the wing motion takes one convective time, (i.e. the free stream flow travels one chord length during the wing motion), and the "slow pitch case" in which the wing motion takes six convective times. The pitch rate during the wing motion is constant. The relevant non-dimensional parameter is the reduced pitch rate

$$
\mathrm{K}=\frac{\dot{\alpha} \mathrm{c}}{2 \mathrm{U}},
$$

where $\dot{\alpha}$ is the pitch rate in radians per second, $\mathrm{c}$ is the wing chord and $\mathrm{U}$ is the free stream speed in consistent units. The values of the reduced pitch rate for the fast and slow pitch cases are 0.39 and 0.065 , respectively. The wing pitching motion history is smoothed at the beginning and end of the constant pitch rate region using the smoothing function proposed be Eldredge et $a l^{2}$ which adapted to the present kinematics is

$$
\alpha=\frac{\alpha_{\max }}{2}\left(\frac{1}{\mathrm{~b}} \ln \left\{\frac{\cosh [\mathrm{b} \mathrm{t} / \Delta \mathrm{t}]}{\cosh [\mathrm{b}(\mathrm{t}-\Delta \mathrm{t}) / \Delta \mathrm{t}]}\right\}+1\right)
$$

where $\alpha_{\max }$ is the maximum pitch angle, $45^{\circ}$ in all cases, $\Delta \mathrm{t}$ is the duration of the pitch motion, and $\mathrm{b}$ is a smoothing parameter. In Equation (2) the start of the pitch motion is a $t=0$ and the end is at $t=\Delta t$.

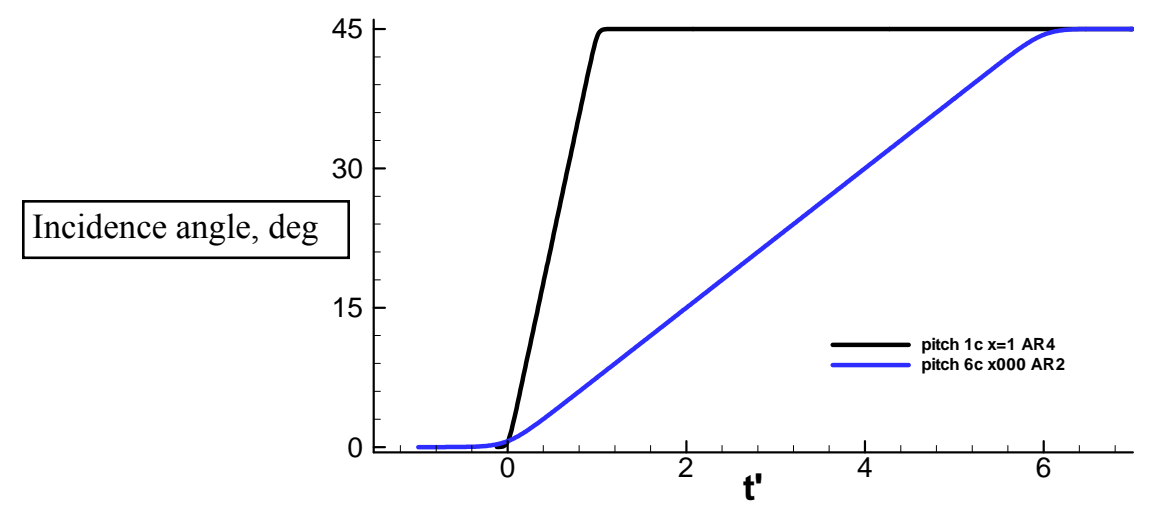

Figure 1. Incidence angle as a function of time. Time is normalized by the flow convective time $t^{\prime}=t c / U$

The AVT 202 panel considered two cases for the pitching kinematics. In one case, the wing is in a free stream or moves at constant speed, U. This case is called here the "translating pitch case". In the other case, the wing rotates at constant angular velocity, the "rotating pitch case". The wing planform is rectangular in both cases with aspect ratio 4 for the translating pitch case and aspect ratio 2 for the rotating pitch case. In the rotating case the incidence flow speed varies along the span of the wing. The incidence speed at the $75 \%$ radial location is used to define of non-dimensional parameters. For the rotating pitch experiments, the wing was mounted with the inboard edge half chord from the axis of rotation.

\section{Flow Facilities and Experimental Methods}

High rate, high amplitude motions are more readily studied in liquids (water, water/glycerin mixtures and the like) than in air ${ }^{3}$. The ratio of model inertia (the model's physical mass) to fluid-dynamic loads is much lower in liquids than in air, leading to improved signal to noise ratio. PIV particle seeding is also easier in the liquid phase. ${ }^{4}$ Table 1 summarizes the facilities used by AVT 202 team members. It includes experimental facilities and relevant instrumentation, and computational facilities/methods. For a complete description of the facilities, experimental methods and data processing used, the reader is referred to the AVT 202 Final Report. ${ }^{1}$ Direct force measurements in unsteady flow present unique data acquisition and processing challenges. Accurate static and dynamic tare procedures are important to remove contributions to the measured force from varying cg location of the model and the model structural dynamics. Also digital filtering is always required to remove electronic noise and, more importantly, signal components associated with structural response. The filter cut-off frequency is a compromise between retaining the relevant frequency content in the flow and removing as much noise as possible. 
Table 1 Summary of Experimental and CFD Facilities

\begin{tabular}{|c|c|c|c|c|}
\hline Team & Members & Facility & Speed/Turbulence & Data \\
\hline AFRL & $\begin{array}{l}\text { K.O. Granlund and } \\
\text { M.V. OL }\end{array}$ & $\begin{array}{c}\text { Water Tunnel } \\
18 " \times 24 " \times 96 " \text { Test } \\
\text { Section }\end{array}$ & $\begin{array}{c}15-40 \mathrm{~cm} / \mathrm{s} \\
\text { Turb. Int. }<0.4 \%\end{array}$ & $\begin{array}{c}\text { Force, Flow } \\
\text { visualization, } \\
\text { PIV }\end{array}$ \\
\hline AFRL & $\begin{array}{l}\text { P. Mancini and A. } \\
\text { Jones }\end{array}$ & $\begin{array}{c}\text { Water Tunnel } \\
18 " \times 24 " \times 96 " \text { Test } \\
\text { Section }\end{array}$ & $\begin{array}{c}15-40 \mathrm{~cm} / \mathrm{s} \\
\text { Turb. Int. }<0.4 \%\end{array}$ & $\begin{array}{c}\text { Force, Flow } \\
\text { visualization, } \\
\text { PIV }\end{array}$ \\
\hline Aselsan Inc. & C. Gozukara & $\begin{array}{l}\text { CFD }- \text { ANSYS } \\
\text { Fluent } \\
60 c \times 40 c \times 60 c \\
\text { Domain } \\
\end{array}$ & $\begin{array}{c}\text { RANS - Laminar } \\
\text { and k- } \varepsilon \text { turbulence } \\
\text { model }\end{array}$ & $\begin{array}{c}\text { Reynolds number } \\
3600-1000\end{array}$ \\
\hline $\begin{array}{l}\text { Cambridge } \\
\text { University }\end{array}$ & $\begin{array}{l}\text { R. Stevens and H. } \\
\text { Babinsky }\end{array}$ & $\begin{array}{l}\text { Water Towing Tank } \\
0.8 \mathrm{~m}^{2} \text { cross section }\end{array}$ & & $\begin{array}{c}\text { Force, Flow } \\
\text { visualization, } \\
\text { PIV }\end{array}$ \\
\hline $\begin{array}{l}\text { Florida State } \\
\text { University }\end{array}$ & $\begin{array}{l}\text { K. Taira and R. } \\
\text { Jantzen }\end{array}$ & & & $\begin{array}{c}\text { Reynolds number } \\
300\end{array}$ \\
\hline $\begin{array}{c}\text { Istambul Technical } \\
\text { University }\end{array}$ & O. Cetiner, O. Son & $\begin{array}{l}\text { Free Surface Water } \\
\text { Channel } \\
1010 \mathrm{~mm} \times 790 \mathrm{~mm} \\
\text { cross section }\end{array}$ & $10 \mathrm{~cm} / \mathrm{s}$ & $\begin{array}{c}\text { Force, Flow } \\
\text { visualization, } \\
\text { PIV }\end{array}$ \\
\hline $\begin{array}{c}\text { Technical University } \\
\text { of Braunschweig }\end{array}$ & $\begin{array}{l}\text { R. Radespiel, R. } \\
\text { Wokoeck }\end{array}$ & $\begin{array}{c}\text { Eiffel-Type Wind } \\
\text { Tunnel } \\
0.6 \mathrm{~m} \times 0.6 \mathrm{~m} \times 1.5 \mathrm{~m}\end{array}$ & Turb. Int. $<0.1 \%$ & Force \\
\hline $\begin{array}{c}\text { Technical University } \\
\text { of Delft }\end{array}$ & $\begin{array}{l}\text { M. Percin and B. W. } \\
\text { van Oudheusden }\end{array}$ & $\begin{array}{c}\text { Octagonal Water } \\
\text { Tank } \\
\text { Diameter } 0.6 \mathrm{~m} \\
\text { Height } 0.6 \mathrm{~m} \\
\end{array}$ & & $\begin{array}{c}\text { Force, Tomographic } \\
\text { PIV }\end{array}$ \\
\hline $\begin{array}{l}\text { University of } \\
\text { Maryland }\end{array}$ & $\begin{array}{c}\text { A. Jones, P. } \\
\text { Mancini, F. Manar }\end{array}$ & $\begin{array}{c}\text { Tow Tank } \\
7 \mathrm{~m} \times 1.5 \mathrm{~m} \times 1 \mathrm{~m}\end{array}$ & & Force \\
\hline $\begin{array}{l}\text { University of } \\
\text { Michigan }\end{array}$ & $\begin{array}{l}\text { H.-T. Yu, L. } \\
\text { Bernal }\end{array}$ & $\begin{array}{c}\text { Free Surface Water } \\
\text { Channel } \\
0.6 \mathrm{~m} \times 0.6 \mathrm{~m} \times 2 \mathrm{~m}\end{array}$ & $\begin{array}{c}5 \mathrm{~cm} / \mathrm{s} \text { to } 40 \mathrm{~cm} / \mathrm{s} \\
\text { Turb. Int. }<1 \%\end{array}$ & $\begin{array}{c}\text { Force, Flow } \\
\text { Visualization, PIV }\end{array}$ \\
\hline
\end{tabular}

PIV measurements were used to measure vortex location and circulation. The $\gamma_{2}$ vortex detection scheme of Graftieaux et al. ${ }^{5}$ was employed by the Cambridge University Team to identify the position of vortex cores in PIV data (contour centroid method). Using the LEV centre calculated by $\gamma_{2}$ as the epicentre, for each frame, a series of circular contours are defined with increasing radius. The circulation $(\Gamma)$ around each contour is calculated and plotted with respect to contour radius. The Lamb-Ossen radial circulation equation (3) is used

$$
\Gamma=\Gamma_{\infty}\left(1-\exp \left(\frac{-r^{2}}{r_{c}^{2}}\right)\right)
$$

with $r$ the radius of given contour and $r_{c}$ the vortex core radius. A best-fit Lamb-Oseen radial variation in $\Gamma$ (Equation 3.) is applied to the data, whence $\Gamma_{\max }$ can be inferred. The calculated error in $\Gamma$ from the fit of a free Lamb-Oseen vortex is $0.23 \%$. 


\section{Results and Discussion}

\section{A. Aerodynamic Force Histories}

We begin with a plot of lift coefficient histories for the four pitching cases, compared side-by-side as translation and rotation, plotted with the same time-base and axes definitions. The "fast" cases are plotted in two ranges of abscissa: one to emphasize the response when acceleration is nonzero, and a broader range (15 chords-traveled) to show long-term settling. The "slow" cases are all plotted with respect to 5 chords traveled. To reiterate, the rotations are with an $\mathrm{AR}=2$ plate with (primarily) $0.5 \mathrm{c}$ root cutout, while translations are with an $\mathrm{AR}=4$ plate. "Fast" pitch has the acceleratory part occurring in a ramp linear with time over 1c of travel, while the "slow" cases do so over 6c. The linear ramp is smoothed at its endpoints. Pitch motions begin with the plate at zero incidence and conclude with the plate at 45 degrees. Lift data are presented in two sequences; the first zooms in to early-time, emphasizing loads-history during the accelerated portion of the motion, and immediately thereafter. The second covers the entire recording-time, showing the long relaxation-transients after cessation of acceleration, in going towards the "steady state" (or bluff-body) response.

The fast cases in Figure 2 show an initial transient during rotation acceleration, followed by an increase in with time during the constant pitch rate part of the wing motion, and a second rapid change during rotational deceleration at the end of the pitch motion. The lift spike during the rotational acceleration is positive. It is attributed to noncirculatory effect. The wing acceleration result in rapid downward fluid acceleration, which in turn results in high lift coefficients. Depending on the smoothing used, the lift coefficient can reach very large values $(>6)$. The lift coefficient after the initial transients varies approximately linear with time, and therefore pitch angle. The lift coefficient values are large in this case $(\sim 4)$. This is much higher that what can be expected from steady flow estimates. It indicates that rotation rate effects are important in this part of the motion, and that these rotation rate effects are not suppressed by flow separation likely present at the high angles of attack. During rotational deceleration, the lift coefficient is significantly reduced. The lift spikes associated with deceleration are less pronounced than for the initial transient. This is likely to be because the pitch angle is now $45^{\circ}$. After the deceleration transient the lift coefficient is large $\sim 2$. The rotational pitch data in Figure 2 (right) show similar trends and lift coefficient values are comparable to the translational cases.

There is perhaps surprisingly good agreement between the various data sets, for each respective case. Occasionally there is an outlier, likely due to balance signal-to-noise reasons, and tare-procedure; often multiple curves mutually align, with one deviating, especially during the accelerated portion of the motion, but then later coming into mutual consonance. This multi-way validation is encouraging, for it unifies the various data sets despite rather stark differences in types of model installation, blockage, Reynolds number and so forth. In particular, in some cases the plate is suspended vertically in a water tunnel or tow tank, with the load-cell atop of the water line, and the "tip" near the load-cell either piercing the free-surface or terminated with a splitter plate. In other cases the plate is mounted horizontally in the test section, supported by a sting aft of the plate, or midway along its pressureside. The different kinds of blockage and physical obtrusions appear to not have any discernible major effect. 

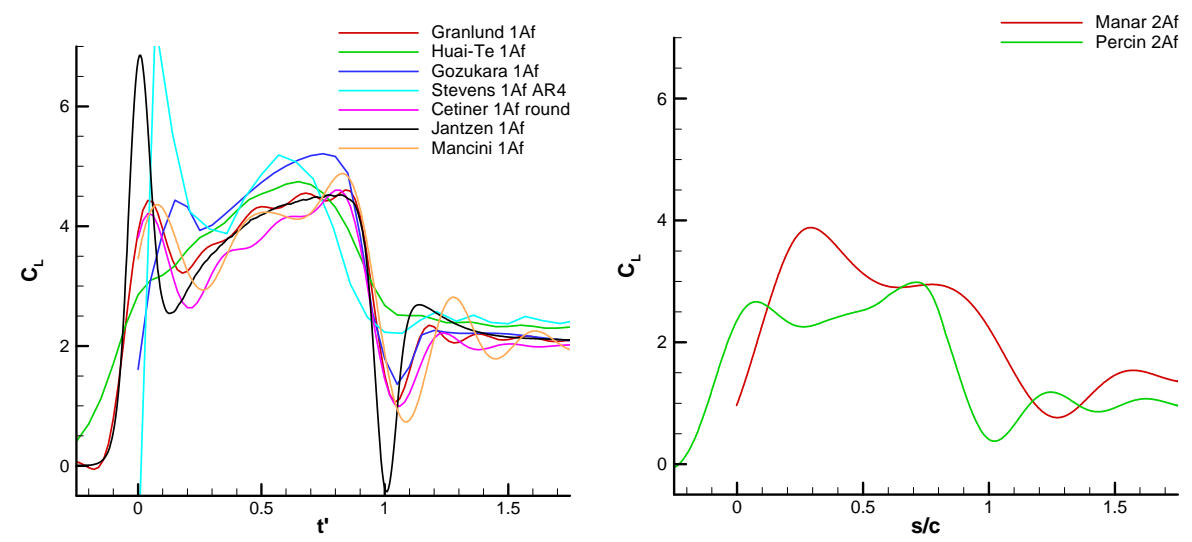

Figure 2. $\mathrm{C}_{\mathrm{L}}$ for the fast pitching cases (LE pivot). Translational (left) and rotational (right).

Figure 3 shows the lift coefficient data for the slow pitching cases. The left plot, which includes the translational pitch cases, shows similar trends as the fast pitching cases, but because of the smaller pitch rate and pitch acceleration the lift coefficient values are significantly lower. In these cases the maximum lift coefficient is $\sim 2$ at the end of the pitch motion. The rotational pitch case in Figure 3 shows similar trends as the translational case, with comparable lift coefficient values.
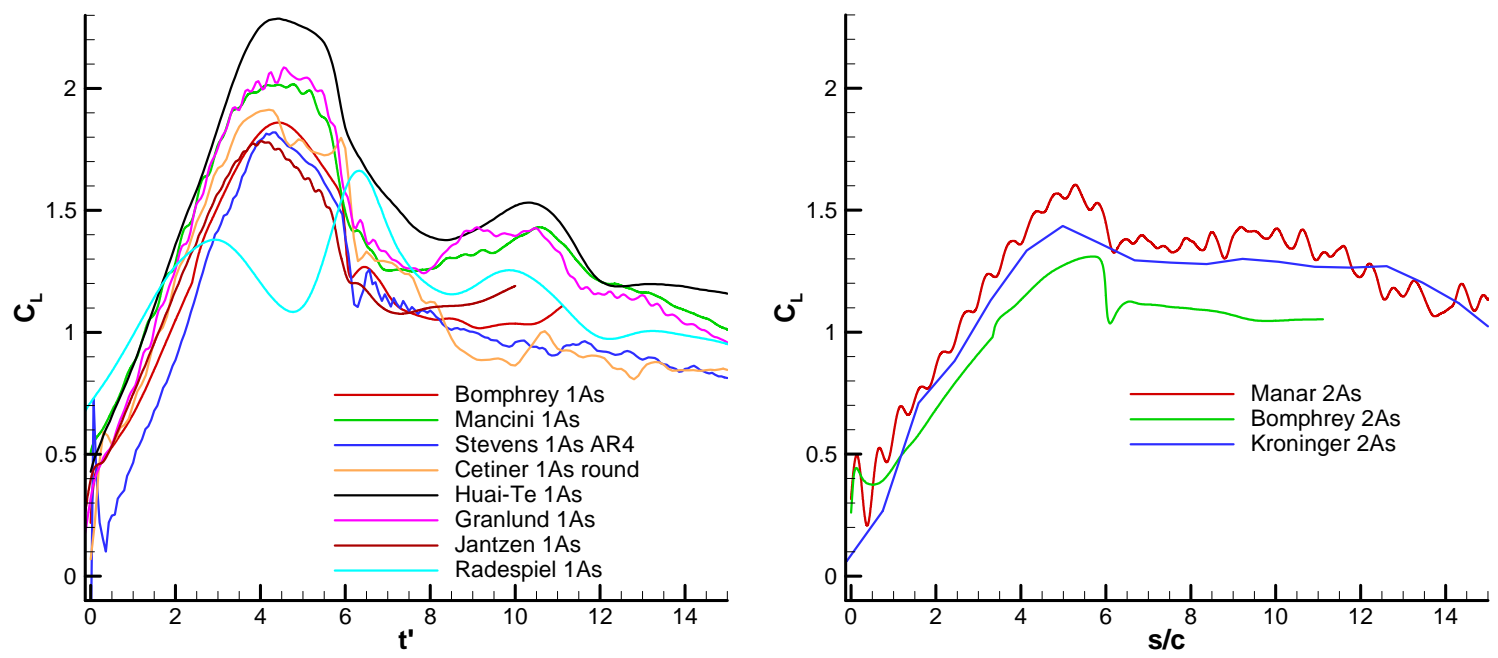

Figure 3. CL for the slow pitching cases (LE pivot). Translational (left) and rotational (right).

The long-term trend shown in Figure 4 for the fast pitch cases is evidently that it takes at least 15 chordstraveled for the effects of acceleration to completely dissipate, and for the lift history to asymptote to the "bluff body shedding" or quasi-steady state. For the translational cases (left plot) there is small local maximum at 7.5 convective times which is indicative of a vortex shedding event. The rotational cases are different: there the lift response reaches a more or less constant value after $5 \mathrm{c}$ of travel, or less. Instead of a long and slow relaxation, there may be a rise in lift after the accelerated portion of the motion is over, followed by a long steady period. In some cases, the wing revolves through its own way, and depending on the blockage of the installation (distance between wing tips and walls of the $\operatorname{tank}^{6}$ ), lift history declines slightly after a full revolution has been completed. 

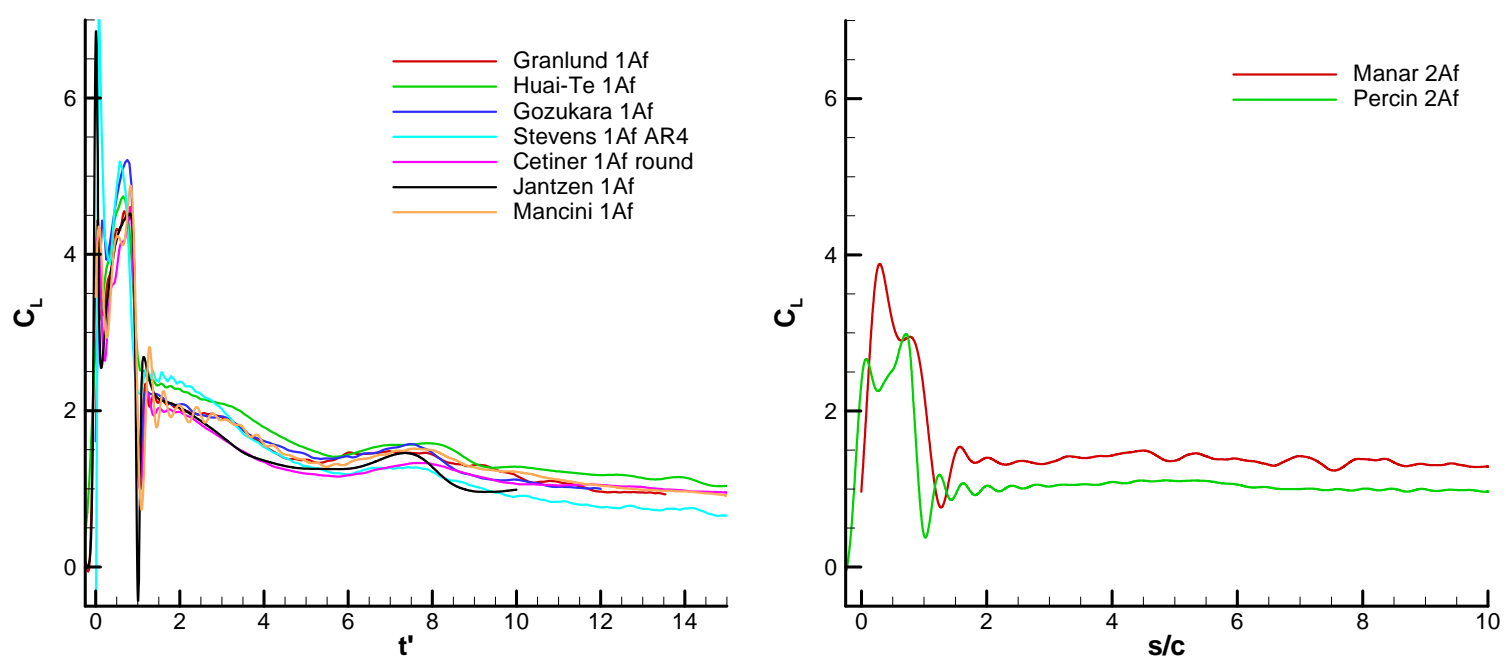

Figure 4. Long-term history of $C_{L}$ for fast pitching cases; translation (left) and rotation (right).

The drag histories, plotted across 15 convective times, for the fast and slow pitching cases are shown in Figure 5 and Figure 6, respectively. In each figure the translational cases are shown on the left and the rotational cases on the right. The drag coefficient evolution follows the same trends as the lift coefficient. In these cases, the force transient due to pitch acceleration at the start of the motion is not present because the wing is aligned with the flow, and pitch deceleration transient at the end of the motion is much more pronounce. The drag coefficient is larger at the end of the motion, and relaxes to the bluff-body value after approximately 15 chords travel. A local maximum is observed at 7.5 chords travel for the fast translational pitch case that suggests a transient vortex-shedding. The rotational cases in the show similar $C_{D}$ behavior as for $C_{L}$ with the coefficient values reaching steady state after only 5 chords travel. The slow translational pitch case in Figure 6 right show a local maximum at $7 \mathrm{c}$ which is somewhat later than for the fast translational pitch indicating differences in vortex shedding for the fast and slow translational pitch cases.
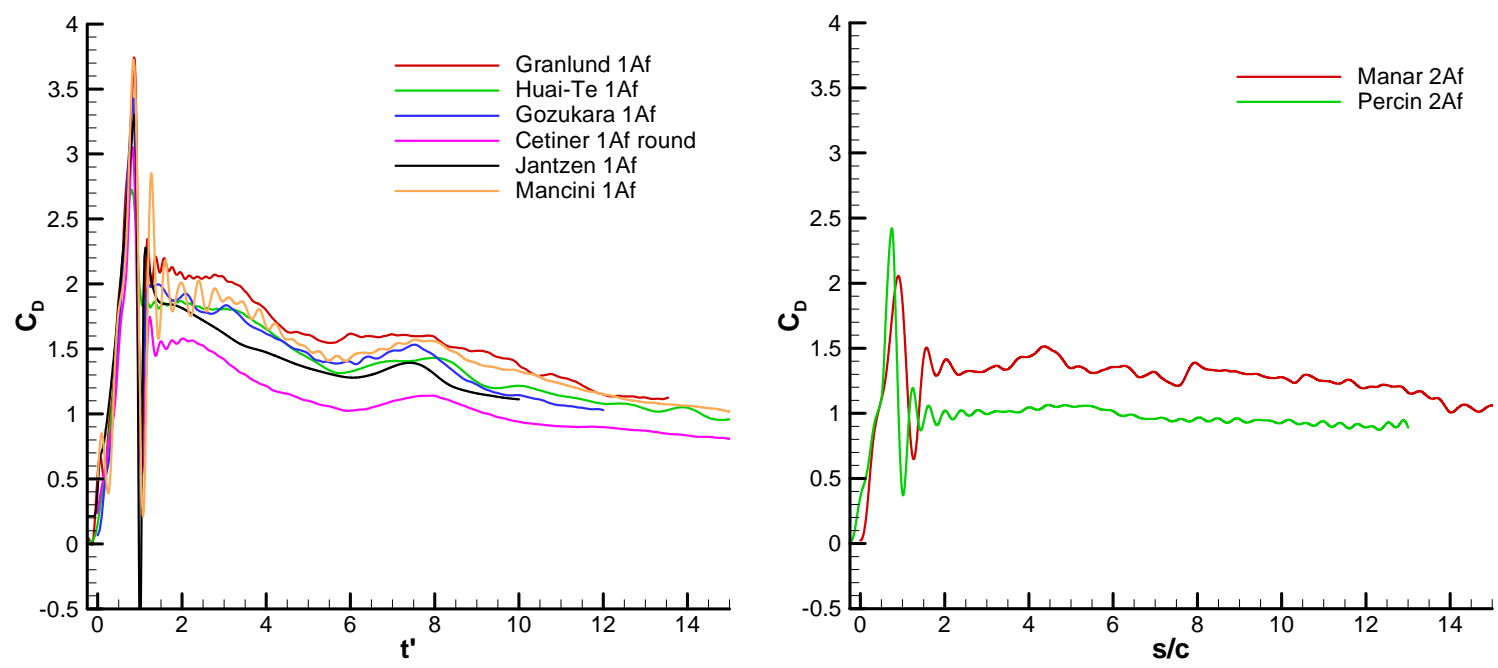

Figure 5. $C_{D}$ for the fast pitching cases. Translational (left) and rotational (right). 

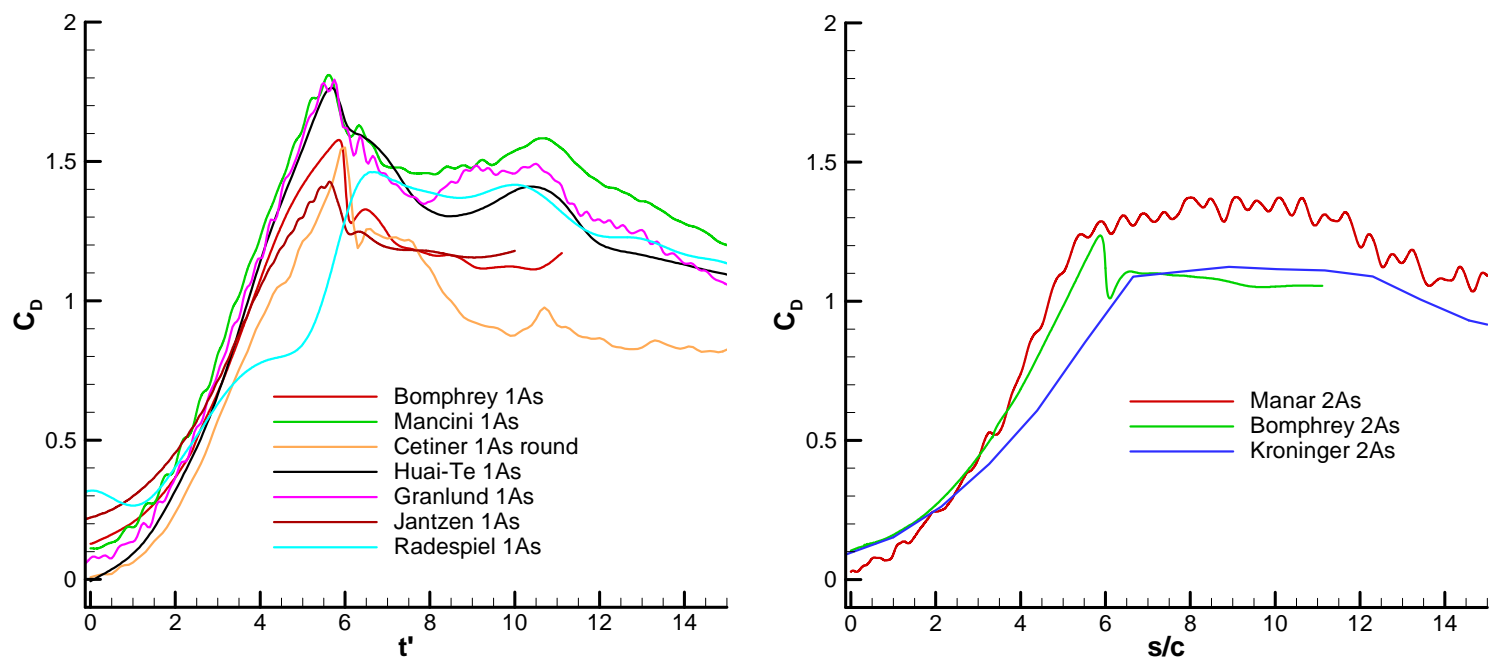

Figure 6. $C_{D}$ for the slow pitching cases. Translational (left) and rotational (right).

\section{B. Evolution of Velocity and Vorticity Fields}

Flow visualization and PIV results are discussed to qualitatively relate LEV, TEV and tip-vortex development to the history of lift and drag. In some cases we compare qualitative and quantitative visualization; that is, dye concentration vs. measured or computed vorticity. LEV and tip vortex (TV) development during pitching is given in Figure 7 (a), (c), (e) and (g); the left-half of the page is for the fast case, and the right-half is for the slow case.

We first consider the pitching fast-case in Figure 7 left images. Initially, the flow is attached with little discernible tip influence at $\mathrm{s} / \mathrm{c}=0.167$. By s/c $=0.5$ a clear and distinct LEV and TV are beginning to form, but with no obvious interaction. As the incidence angle increases $(\mathrm{s} / \mathrm{c}=0.833$ and $\mathrm{s} / \mathrm{c}=1)$ the TV grows concurrently with the LEV. The angle between the distinct LEV and TV from the LE and tip apex is approximately $45^{\circ}$. The downwash from each vortex will superimpose here, and this mechanism may point towards a physical reason as to why the LEV remains more coherent closer to the wing tip. During the translation part of the motion (Figure 7 (b), (d), (f) and (h)), the dye disperses as the LEV and TV grow. By s/c = 5, the dye from the LE is fully entrained into the tip vortex and by $\mathrm{s} / \mathrm{c}=8$ we see the beginning of the reformation of an LEV type structure in the outer span region of the wing, albeit a much less defined structure than that which manifested while pitching occurs.

Turning to the slow pitching case (right-hand side of Figure 7), initially the flow evinces small and well-defined TV and LEV. By s/c = 3, the TV has developed but remains coherent, whereas the LEV is weak and only has a coherent form close to the tip. At $\mathrm{s} / \mathrm{c}=5$ the LEV has dispersed, and by $\mathrm{s} / \mathrm{c}=6$, the tip vortex is the dominant structure. After cessation of pitching motion, the flow is largely separated, with the exception of the reformation of a small LEV-type structure at $\mathrm{s} / \mathrm{c}=10$. Comparing again with the lift coefficient history, we gain some physical insight and propose that the presence of a weak LEV/weak circulatory flow is intrinsically linked to an increase in streamline curvature, and is therefore responsible for an increase in the force. 


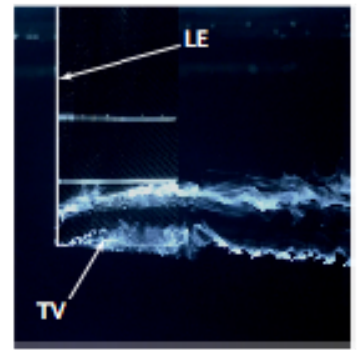

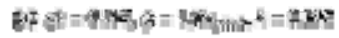

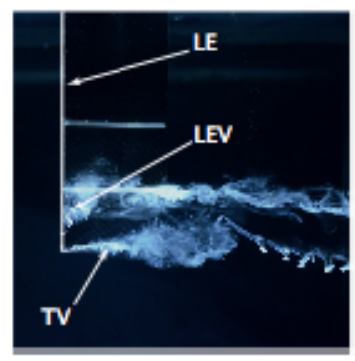

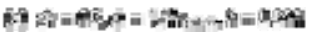

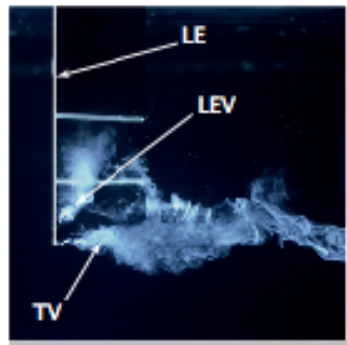

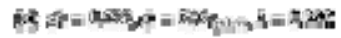

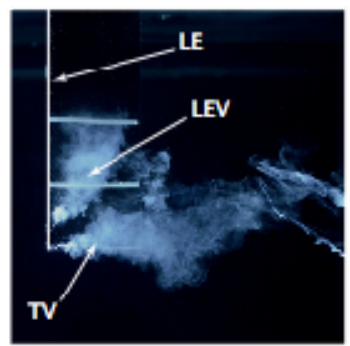

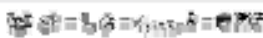

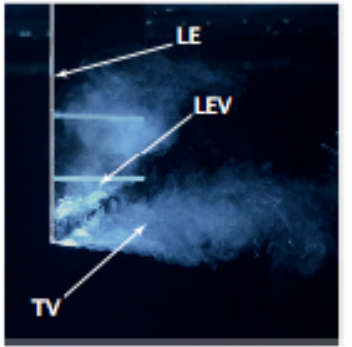

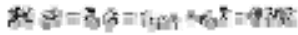

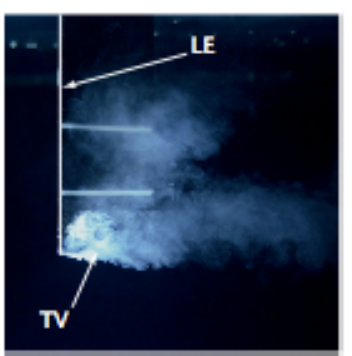

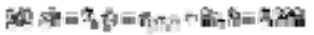

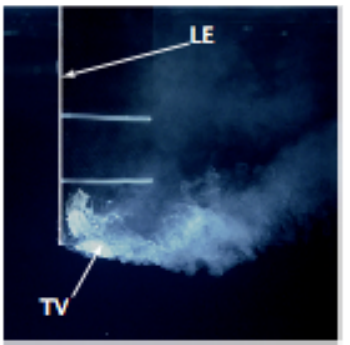

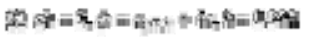

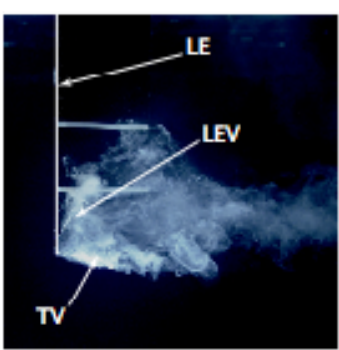

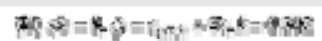

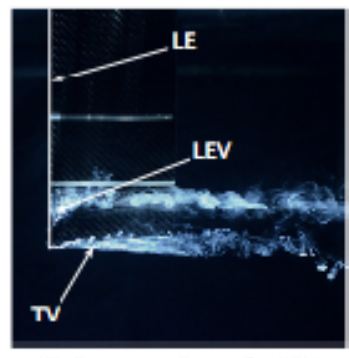

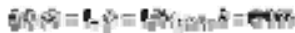

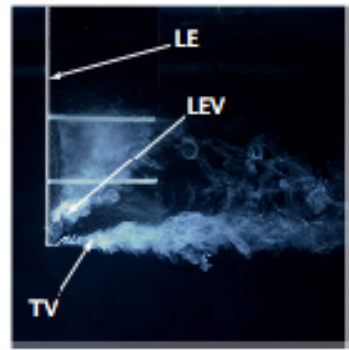

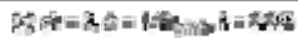

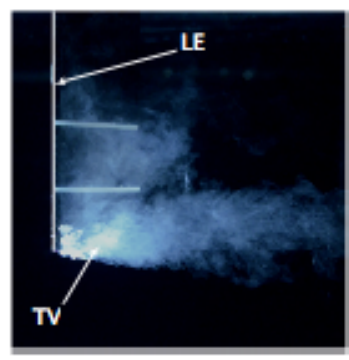

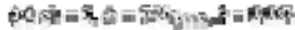

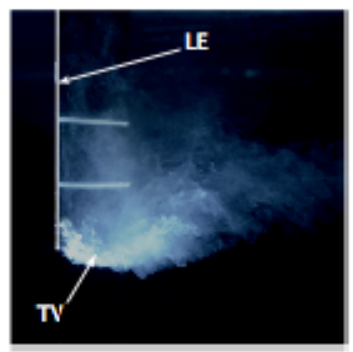

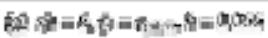

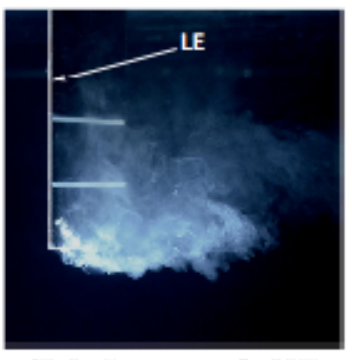

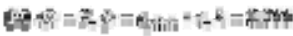

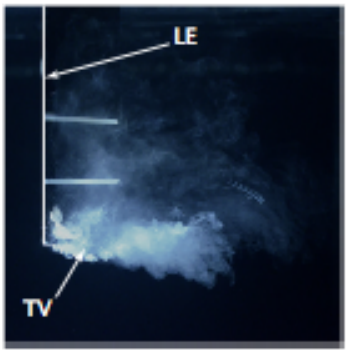

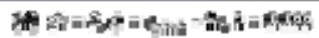

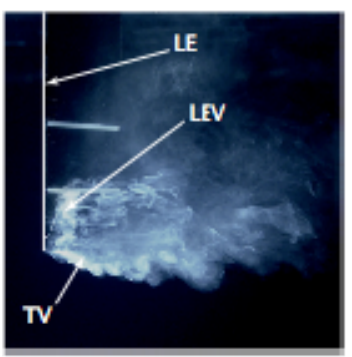

A9 s.

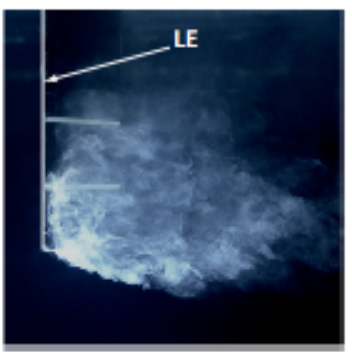

fin

Figure 7. Translational pitch, LEV/TV interaction flow visualization; eight images on left are for the fast case (1 chord pitch), and eight images on the right are for the slow case (6 chords pitch). Instances of chordstraveled as marked, progressing sequentially for the fast case down the first column of the figure, then the second column; and for the slow case down the third column of the figure, and then down the fourth column.

We now turn to an alternative presentation of the fast pitching case, as an isometric view of sectional visualizations. Fluorescent rhodamine dye injection illuminated by a planar light sheet is compared with black and white rendition of computed vorticity contours in Figure 8. Evidently a "suitable" value of normalized out-of-plane vorticity value renders the quantitative vorticity information looking very similar to the qualitative dyeconcentration information. 

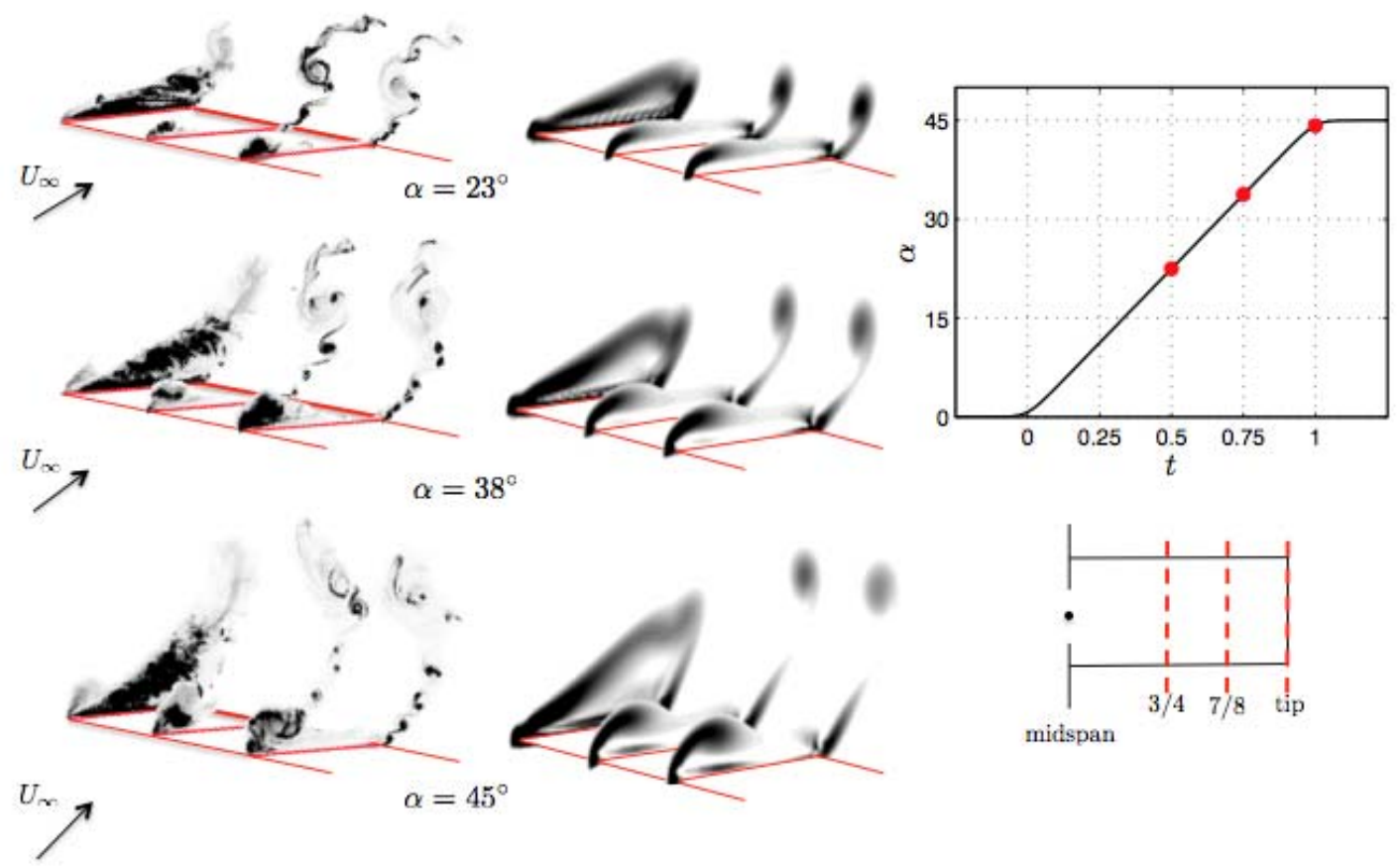

Figure 8. Juxtaposition of flow visualization by planar laser illumination of fluorescent dye in a water tunnel at $\mathrm{Re}=20,000$ (left column) and black and white rendition of spanwise vorticity contours from direct numerical simulation at $\operatorname{Re}=\mathbf{3 0 0}$ (middle column) for the fast translational pitching case. Instances of flow evolution along the motion-history as marked, visualized at the $3 / 4$ span, $7 / 8$ span and tip locations.

\section{Conclusions}

1. Whether rotational or translational, the pitching cases produce a much larger lift than the fast surging cases. This is the pitch-rate effect. After the pitching motion has concluded, there is a large and fast drop in lift, followed by slow relaxation to the "steady state" (rotation) or bluff-body response (translation). The two fast pitching cases are very similar in lift history, as are the two fast surging cases.

2. Depending on choice of normalization, one may find the long-term value of lift for the rotational motion to be larger than for the translational motion, or smaller. But it does appear that for the rotational motion the lift asymptotes to a constant value, presumably because of a spatially and temporally more organized flow (by some reckonings a more stable LEV, especially near the wing root) on the plate's suction side, that differs from the bluff-body solution, which Karman-sheds or just has a dead-water region.

3. For fast motions, the peak lift occurs at approximately the point where acceleration concludes. For the slow cases, lift peaks at some earlier time, before the surging motion or the pitching motion is complete, in a stalllike process where the LEV grows, reaches saturation and sheds, all while acceleration of the plate proceeds. The shedding effect is mediated by rotation. One supposes that the slower motions are governed by a vortex formation-time phenomenon, presumably captured in vortex dipole ideas, while for the faster motions, LEVs are still growing and accumulating vorticity at motion's end.

4. Secondary peaks in lift, several convective times after acceleration is over, are observed in some cases. These are presumably due to formation of new LEVs, and appear to depend on plate aspect ratio (and installationeffects in experiments).

5. It takes many convective times ( 15 or more for translation, perhaps 5 for rotation) to reach the steady-state lift value.

6. For translational fast pitch and translational fast surge, the LEV initially convects away from the LE at approximately one third to half of the reference free-stream speed, while the TEV convects at close to the full 
free-stream speed. This gives a vortex-force resembling $\rho \mathrm{U} \Gamma$. Exact speeds DO vary from case to case, and accuracy is important if we're going to properly capture the total lift force.

7. Vortices are more coherent with increasing reduced-frequency. Individual vortices are harder to identify for the $6 \mathrm{c}$ cases. The flow is also more 3D for slower cases. 3D effects take time to develop. But even massively 3D cases evince possibility of description by $2 \mathrm{D}$ reduced-order model in a strip-theory sense. In any case, pitch is kinematically dominated (apparent-mass and Magnus effect), while surge is more vortex-dominated (time rate of change of LEV-TEV dipole impulse).

\section{Acknowledgments}

The author would like to thank Dr. Michael Ol (Air Force Research Laboratory) and Profs. Holger Babinski (Cambridge University) and Anya Jones (University of Maryland), as well as, all the members AVT 202 Panel for many fruitful discussions. The data presented here is actually their contribution and I borrowed liberally from the many documents and the AVT final report in preparing this paper. The work at the University of Michigan was sponsored in part by the Air Force Office of Scientific Research's Multidisciplinary University Research Initiative (MURI) under Contract FA9550-07-1-0547, by the Michigan/AFRL Collaborative Center in Aeronautical Sciences.

\section{References}

1 “AVT-202: Extensions of Fundamental Flow Physics to Practical MAV Aerodynamics" Final Report

${ }^{2}$ Eldredge, J. D., Wang, C., and Ol, M. V. "A Computational Study of a Canonical Pitch-up, Pitch-down Wing Maneuver," AIAA Paper 2009-3687, June 2009.

${ }^{3}$ OL, M.V., Parker, G., Abate, G., and Evers, J. "Flight Controls and Performance Challenges for MAVs in Complex Environments". AIAA 2008-6508.

${ }^{4}$ Raffel, M., Willert, C.E., Wereley, S.T., and Kompenhans, J. Particle Image Velocimetry: A Practical Guide. Springer, 2007.

${ }^{5}$ Graftieaux, L., Michard, M., and Grosjean, N. "Combining PIV, POD and vortex identification algorithms for the study of unsteady turbulent swirling flows". Meas. Sci. Technol., Vol. 12, No. 9, August 2001.

${ }^{6}$ Manar, F., and Jones, A.R. "The Effect of Tip Clearance on Low Reynolds Number Rotating Wings". AIAA 20141452. 